\title{
Network Topology Measurements Research Based on Campus Networks
}

\author{
Hui-jie $\mathrm{Qu}^{1, \mathrm{a}}$, Yan Huang ${ }^{2, \mathrm{~b}}$, Dan Zhao ${ }^{1, \mathrm{c}}$ and Zhi Zhao ${ }^{1, \mathrm{~d}, \star}$ \\ ${ }^{1}$ School of Mathematics and Information Science, Guangxi College of Education, Nanning 530023, \\ China \\ 23-306, No.168 Mawangdui Road, Changsha 410016, China \\ aqq_rr252@126.com, ${ }^{\mathrm{b}}$ asd_2006@163.com, ${ }^{\mathrm{c}} \mathrm{zh}$ aodan0519@163.com, ${ }^{\mathrm{d}} \mathrm{zz191} @ 126 . c o m$ \\ ${ }^{*}$ Corresponding Author: Zhi ZHAO
}

Keywords: Topology measurement, FDB, SNMP.

\begin{abstract}
With the constant expansion of campus networks, their architectures have become more and more complicated. Thus, effective network management has become critical for normal operations of networks. It is of great theoretical and realistic research significance for effectively measuring network topology according to demands for managing campus networks. In this paper, a prototype testing system is designed for topology measurements with FDB-based algorithms for measuring link layer topology. With functions for measuring topology and displaying topological graphs, this system is demonstrated to be effective for implementing these two functions under experimental conditions.
\end{abstract}

\section{Introduction}

After their construction and application, how to effectively operate and scientific manage campus networks has become a problem that managers of these networks expect to solve.

As an important function of network management, network topology measurement is performed for the major purpose of acquiring and maintaining data about existence of network nodes and their connections, based on which topology of the whole network is designed. Network managers may detect network faults and discover network bottlenecks according to topological data, so as to optimize an entire network.

According to network layers, topology measurements are divided into third-layer (network layer) and second-layer (data link layer) measurements. Campus networks are typical switched Ethernet, where the second layer of a 7-layer OSI (Open System Interconnection) model, namely the data link layer, is the major layer that corresponds to devices. The key for topology measurement on the data link layer is inferring interconnections between switches.

Techniques for topology measurement mainly cover two steps for making topology measurements. First of all, certain algorithms for topology measurements are adopted to detect all network devices and identify their interconnections. Next, topology graphs are created and displayed on graphical user interfaces with graphical display techniques and graph updating strategies. Hence, algorithms for topology measurements play decisive roles in techniques for performing these measurements.

\section{Design of FDB-based Algorithms for Measuring Link Layer Topology}

In general, algorithms for measuring datalink layer topology are firstly used for detecting all 
switches of a target network by MIB-II (RFC-1213) [1]. Next, data about switches and forwarding database (FDB) are acquired from Bridge MIB (RFC-1493) of each switch. Subsequently, physical topology measurements are performed to identify interconnections between switches, and general nodes are allocated to corresponding switches.

Based on the SNMP (Simple Network Management Protocol), forwarding databases of devices on the datalink layer are acquired. In the next step, interconnections between switches are deduced according to information regarding the forwarding databases. Yuri Breitbart[2] et al proposed a theory and algorithm known as lemma on direct interconnections for inferring interconnections between switches in a heterogeneous network. Based on previous theories, Bruce Lowercamp[3] et al performed more intensive studies and put forward Lemma4 (Concerning Simple Interconnections): $S_{i k}$ is simply interconnected with $S_{j l}$ only when $T_{i k} \mathrm{I} T_{j l}=\varnothing$.

To further lower the standard for forwarding databases for topology detection, judgment rules for direction interconnections and those for interconnection with dummy devices were proposed based on Bruce Lowercamp’s lemma for simple interconnections [4].

\section{Judgment Rules for Direct Interconnections}

Rule 1: It is assumed that the switch is $R$ at Port $a$ and $A_{R a} \neq \varphi$ only applies to the switch $S \in A_{R a}$, so that all switches can be simply interconnected between $S$ and $A_{R a}-\{S\}$, and $r$ is the port that $S$-oriented root node. Provided that $A_{S r}$ is tenable and only contains $R, S_{a}$ and $S_{r}$ will be directly interconnected.

\section{Judgment Rules for Interconnections between Dummy Devices}

Rule 2: It is hypothesized that Port $a$ exists in the switch $R$ and $A_{R a} \neq \varphi$. If several switches $S_{i}$ belong to $A_{R a}$, in order that switches can be simply interconnected between $S_{i}$ and $A_{R a}-\left\{S_{i}\right\}$, while $r_{i}$ is a port at the $S_{i}$-oriented root node. Assuming that I $A_{\text {Siri }}>\{R\}$, the dummy devices $Y$ and $R$ will be directly interconnected. At the same time, the switch $A_{\text {siri }}-\{R\}$ and $Y$ will be directly interconnected.

FDB-based algorithm is designed for measuring link layer topology according to the lemma on simple interconnections (Lemma 4), Rule 1 and Rule 2.

The information necessary for topology inference is stored in each switch, and to realize the algorithm, two queues are employed, including one queue of nodes to be detected, which consists of intermediate nodes of the current spanning tree, and the other queue of nodes to be generated, which is made up of nodes of leaf switches in the current spanning tree. The algorithm is just implemented to find out interconnections of objects between the queues of nodes to be generated and detected. The algorithm for acquiring topology related information from switches is described as follows from the perspective of pseudocodes:

(1) Acquire databases from all switches inside subnets;

(2) All switches inside Ping subnet are utilized for filling up buffer of forwarding databases of all switches inside subnets; 
(3) Forwarding databases of switches are read in succession;

(4) A set of uplink and downlink ports is created for each switch in its forwarding database. Meanwhile, nodes of all switches inside subnets are included in the queue to be detected;

(5) Nodes of switches are successively pushed into the queue of nodes to be generated, which is an FIFO (first in, first out), and leaf switches are removed from the queue of nodes to be detected;

(6) A node is taken from the queue to be generated for detection;

(7) Check whether the MAC (media access control) address is included in the forwarding databases of all ports in the sets of downlink ports of other nodes to be detected. Provided that the address is searched in any database but there is more than one node, the database shall be deleted. If only the node to be detected is searched, the corresponding port number will be directly interconnected with the uplink port of the node to be detected, and the port shall be removed from the set of downlink ports at the current node;

(8) Once a node is traversed and the set of its downlink ports is discovered to be empty, the nodes of the corresponding switch shall be pushed into the queue of nodes to be generated and simultaneously removed from the queue of nodes to be detected;

(9) Repeat steps 6 to 9 if the set is not empty in the queue of nodes to be generated.

\section{Design of a Prototype Testing System for Topology Measurement}

According to the FDB-based algorithms for measuring datalink layer topology designed above, a prototype testing system is designed for topology measurement by SNMP++ development kit and $\mathrm{VC}++6.0$, to display the topological structure acquired from topology measurements on graphic interfaces.

\section{Analysis of System Requirements}

Topology measurement is performed for the ultimate purpose of providing correct topological structures for network management and displaying them in front of managers on graphic interfaces, in order to effectively monitor network devices and lines. Therefore, the system is required to not only effectively measure topological structures by appropriate algorithms for topology measurements, but also to completely display these structures on graphic interfaces.

\section{System Architecture}

Based on its run modes, a network system may comprise of three layers. Being essential for topology discovery, the bottom layer contains topological data. On this layer, the module for topology measurement is a basic unit of work for measuring topology. It takes charge of acquiring topological data from networks within certain scope and subsequently transmits the data to the intermediate layer in some forms.

Used for analyzing topological data, the intermediate layer is mainly available for analyzing and judging the data.

The top layer is a topological data presentation layer, which is responsible for topology display and inter-operations with users.

The architecture of the system is shown in Fig. 1 as follows. 


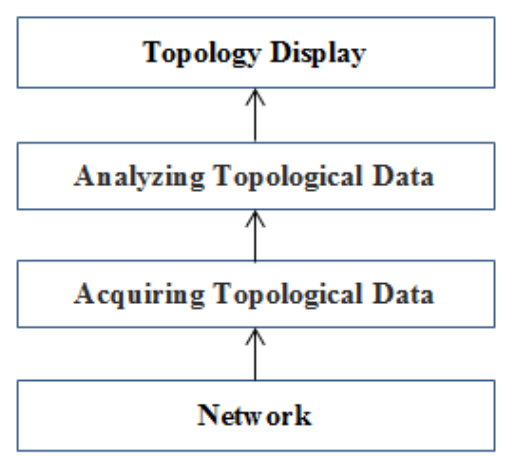

Fig. 1 Architecture of the Measurement System

\section{Design of Functional Module}

According to its functions, the entire system may be divided into several parts, as shown in Fig. 2.

(1) Functional module for master control

This module is mainly used for initializing programs and releasing system resources at the end of programs.

(2) Graphical user interface

It is a window-oriented graphical interface that covers the entire system.

(3) Module for measuring topological data

A designated device is employed for traversing all switches of the network, to acquire complete data about network connections.

(4) Module for displaying topological graphs

The topological data acquired about connections are transformed into specific topological graphs.

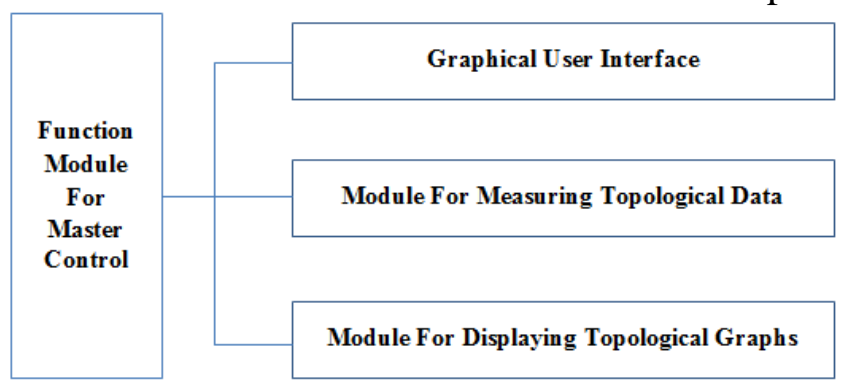

Fig. 2 Module Structures

\section{Tests and Experimental Results}

\section{Experimental Conditions}

A network was built with Huawei S5700, Ruijie RG-S3760 and Cisco WS-C2960 switches in a laboratory. Widely used within campus networks, these switches are supplied by well-known mainstream equipment suppliers, so these devices are typical and representative.

\section{Testing Schemes}

1) Huawei S5700 is used as root node, which is as effective as an aggregation layer switch; Ruijie RG-S3760 and Cisco WS-C2960 are employed as leaf nodes, which are connected to the Huawei switch just like access layer switches. The topological structure of the Testing Scheme 1 is shown in Fig. 3 as follows. 


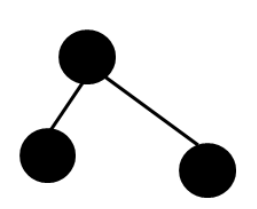

Fig. 3 Topological Structure of Testing Scheme 1

In this testing scheme, six ports on the switch of root nodes are connected to the Ruijie switch, while 18 ports are respectively connected to the Cisco switch to verify if the system is effective for judging direct interconnections of switches.

2) Huawei S5700 is utilized as a root node, which is functionally equivalent to the switch on the core layer; Port 24 of Ruijie RG-3760 switch is an uplink port, which is accessed into six ports of the Huawei switch to perform functions like an aggregation layer switch; Port 24 of the Cisco WS-C2960, as an uplink port, is connected to the Ruijie RG-S3760 switch to function like an access layer switch. The topological structure of the Testing Scheme 1 is shown in Fig. 4 as follows.

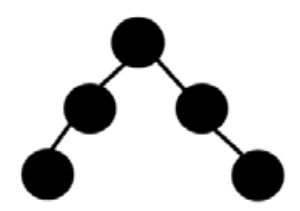

Fig. 4 Topological Structure of Testing Scheme 2

In this scheme, two switches are interconnected with each other in succession. The root node is directly interconnected with the intermediate switch, so is the intermediate switch and the leaf node. However, the leaf node is indirectly interconnected with the root node. Both direct and indirect interconnections exist, which further verifies that the system is useful for accurate judgment of direct and indirect interconnections between switches.

\section{Test Results and Analyses}

The measurement of the testing system starts from the root node. Fig. 5 shows initial setting of a switch for a system.

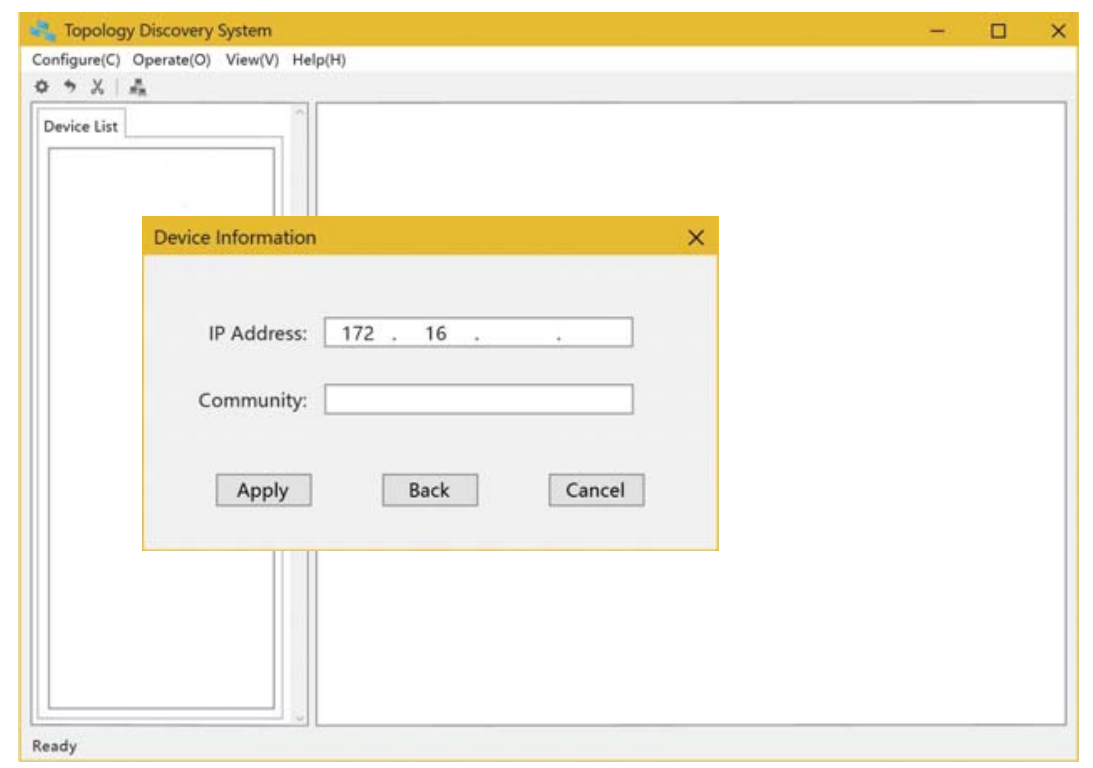

Fig. 5 Interface for Initial System Setting 
1) The measurements of Scheme 1 are displayed.

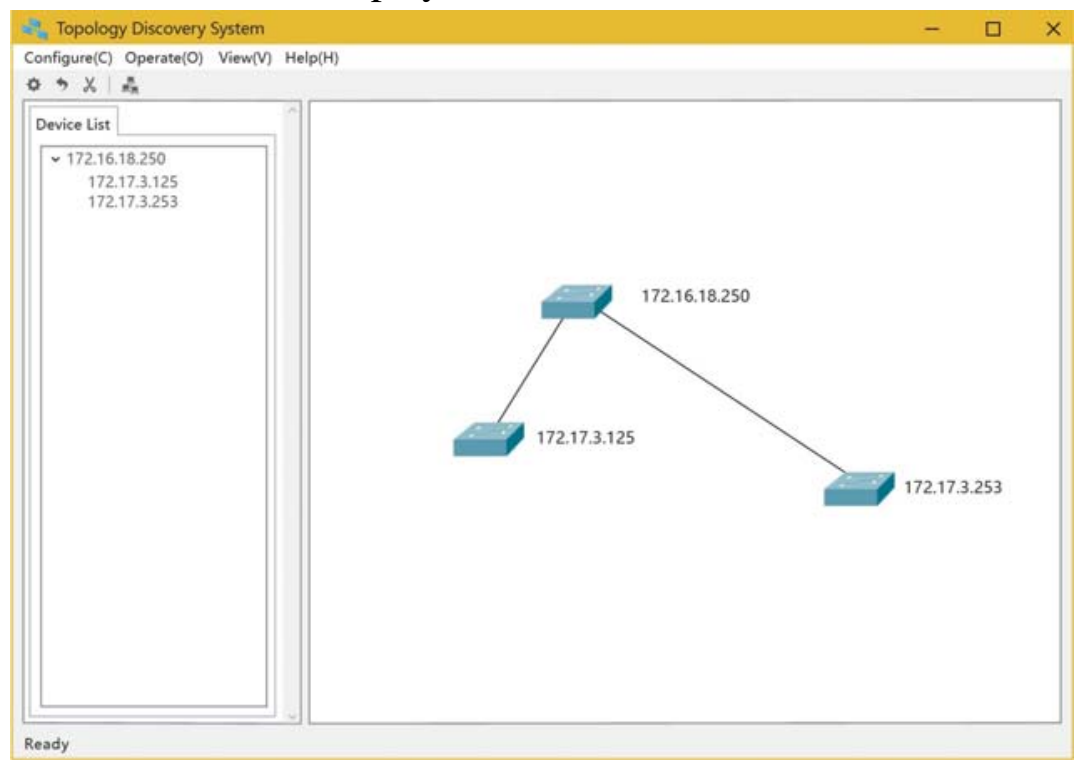

Fig. 6 Measurements of Scheme 1

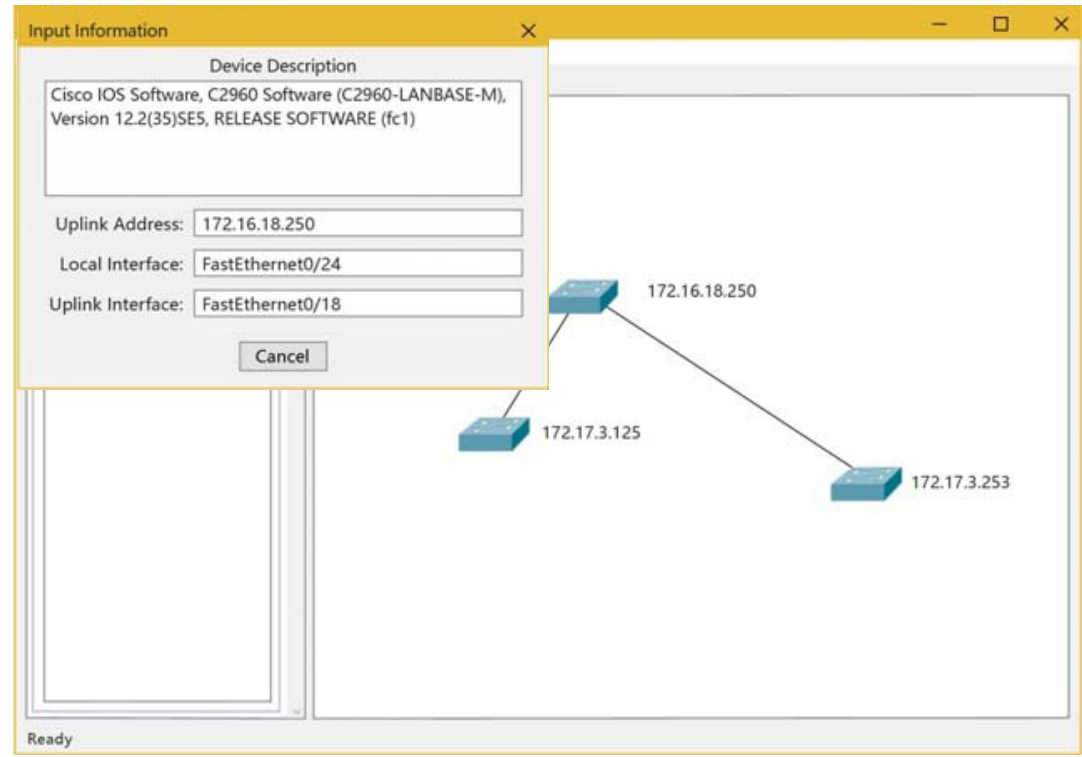

Fig. 7 Results of Ports Corresponding to Topology Measurements of Scheme 1

Result analysis: This is a typical direct interconnection in a topological structure, where switches at two leaf nodes are directly connected to the root switch.

(1) According to measurements in Fig. 6, interconnections of switches are in line with the actual topological structure;

(2) Fig. 7 shows read information about descriptions of the Cisco switch. As shown in Fig 7, the equipment 172.17.3.253 is a Cisco switch with Port 24 as its uplink port, which is connected to Port 18 of the Huawei switch (172.16.18.250). This is consistent with actual port connections of switches established under the experimental conditions.

2) The Measurements of Scheme 2 are displayed. 


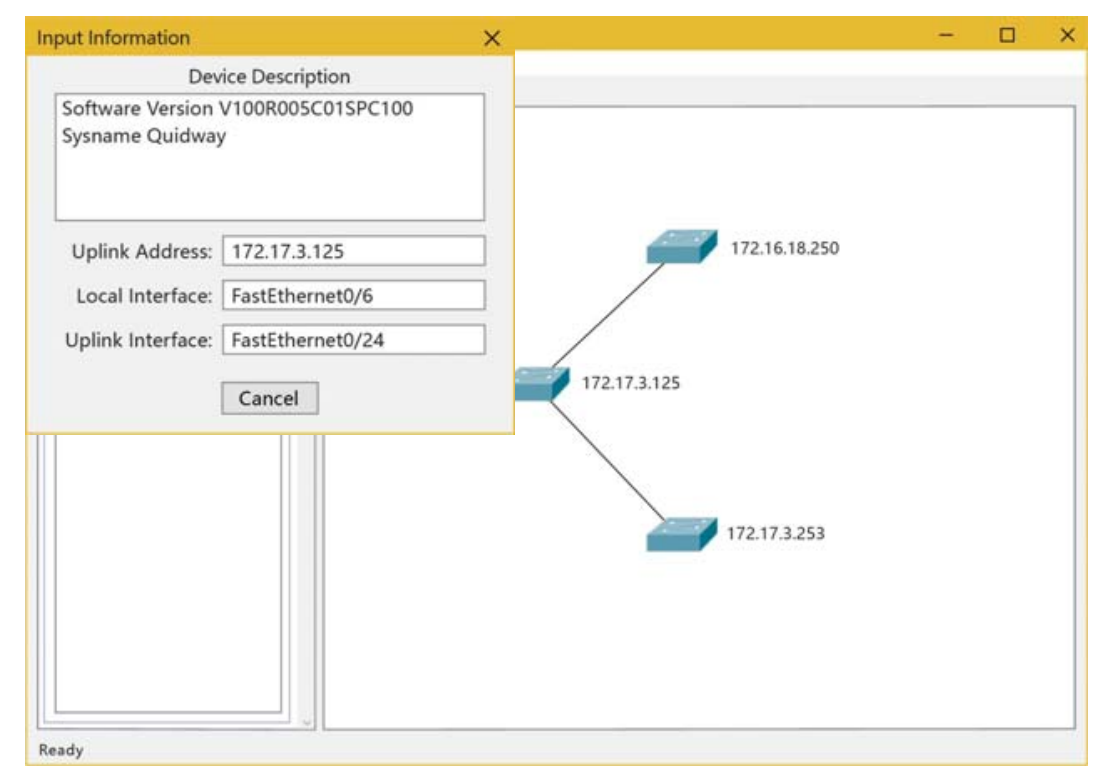

Fig. 8 Topology Measurements of Scheme 2

Result analysis: In Scheme 2, switches are partially directly and indirectly interconnected. The leaf nodes are indirectly connected with the root node. The test results in Fig 8 suggest that the module for topology measurements can accurately discriminate two pairs of direct-connected switches and indirect-connected ones. The read information about descriptions of the equipment 172.16.18.250 shows that Port 24 is the uplink port of the Ruijie switch located in 172.17.3.125 and in Port 6 of the Huawei switch, which is in accordance with the actual interconnections between switch ports and may correctly reflect their relationships.

\section{Summary of Experimental Results}

The results of above tests suggest that the prototype testing system is quite effective for detecting all switches and correctly displaying them on a graphical interface with FDB-based algorithms for datalink topology measurements and various experimental schemes, which is in line with the actual topological structures constructed under the experimental conditions. In addition, port interconnections between switches also tally with corresponding practical connections, so expected purposes of experimental tests have been realized.

\section{Conclusions}

Topology measurements are important for network measurements, while linklayer topology measurements shall meet higher standards for granularity. The prototype system designed in this paper is just an initial one for topology measurements for the major purpose of verifying algorithms for these measurements and making it possible to display topology in a graphical interface. As a complete practical system, it is expected to be further investigated and designed in combination with database and GIS technologies, in order improve storage of topological graphs and dynamic update strategies for these graphs.

\section{Acknowledgment}

This research was financially supported by the Education Department of Guangxi Province (NO.2015JGZ172), and scientific research projects of Guangxi College of Education in 2015 (NO. A2015002), and 2015 Special Program on Hybrid Teaching Reform for Education and Teaching Reform of Guangxi College of Education (NO.XJJG15A03) , and 2016 
Program about Teaching Teams with “Key Competencies” in Guangxi College of Education, Topic: Research on Integrating Cultivation of Key Competencies into Teaching.

\section{References}

[1]K.McCloghrie,M.Rose,RFC 1213, Management Information Base for Network Management of TCP/IP-based internets: MIB-II, March 1991.

[2]Y.Breitbart,M.Garofalakis,C.Martin,R.Rastogi,S.Seshadri,A.Silberschatz, Topology Discovery in Heterogeneous IP Networks,Proc.Of IEEE INFOCOM 1(2000)265-274.

[3]B.Lowekamp,D.R.O’ Hallaron,T.R.Gross. Topology Discovery for Large Ethernet,ACM SIGCOMM (2001).

[4]Zeng-Wang LV, Hua-Ping HU, Bo LIU, Research on Algorithms for Detecting Physical Topologies of Local Area Network, Computer Engineering \& Science. 28(2006) 81-83. 